\title{
Screening stringy horizons
}

\author{
Gaston Giribet $^{1,2,3, a}$, Arash Ranjbar ${ }^{1,4}$ \\ ${ }^{1}$ Université Libre de Bruxelles and International Solvay Institutes, ULB-Campus Plaine CPO231, 1050 Brussels, Belgium \\ 2 Departamento de Física, Universidad de Buenos Aires and IFIBA-CONICET Ciudad Universitaria, Pabellón 1, 1428 Buenos Aires, \\ Argentina \\ ${ }^{3}$ Instituto de Física, Pontificia Universidad Católica de Valparaíso, Casilla 4059, Valparaiso, Chile \\ ${ }^{4}$ Centro de Estudios Científicos (CECs), Arturo Prat 514, Valdivia, Chile
}

Received: 18 August 2015 / Accepted: 1 October 2015 / Published online: 14 October 2015

(C) The Author(s) 2015. This article is published with open access at Springerlink.com

\begin{abstract}
It has been argued recently that string theory effects qualitatively modify the effective black hole geometry experienced by modes with radial momentum of order $1 / \sqrt{\alpha^{\prime}}$. At tree level, these $\alpha^{\prime}$ effects can be explicitly worked out in two-dimensional string theory and have a natural explanation in the T-dual description as coming from the integration of the zero mode of the linear dilaton, which yields a contribution that affects the scattering phase shift in a peculiar manner. It has also been argued that the phase-shift modification has its origin in a region of the moduli space that does not belong to the exterior black hole geometry, leading to the conclusion that at high energy the physics of the problem is better described by the dual model. Here, we elaborate on this argument. We consider the contribution of world-sheet instantons in the two-dimensional Euclidean black hole $\sigma$-model and study its influence on the phase shift at high energy.
\end{abstract}

\section{Introduction}

In the recent paper [1], it has been argued that string theory effects qualitatively modify the effective black hole geometry experienced by modes with radial momentum of order $1 / \sqrt{\alpha^{\prime}}$. At tree level, these $\alpha^{\prime}$ effects can be explicitly worked out in two-dimensional string theory, where the black hole background admits an exact world-sheet description in terms of the gauged $S L(2, \mathbb{R}) / U(1)$ Wess-ZuminoWitten (WZW) model. In addition, this model is well known to have a dual description (in a sense similar to T-duality) that involves a two-dimensional flat tachyonic linear dilaton background, known as Fateev-Zamolodchikov-Zamolodchikov (FZZ) dual [2]. In the FZZ dual model, the $\alpha^{\prime}$ effects studied in [1] have a natural explanation as coming from the integration over the zero mode of the linear dilaton, which yields a

a e-mail: gaston@df.uba.ar contribution that affects the scattering phase shift in a peculiar manner. Such contributions and, consequently, the phaseshift modification they produce seem to come from a region of the moduli space that does not belong to the exterior black hole geometry. This led the authors of [1] to conclude that the high-energy physics is better described by the FZZ dual model, rather than by the two-dimensional (2D) black hole $\sigma$-model. Here, we elaborate on this argument. We consider world-sheet instanton contributions and study their influence to the phase shift at high energy. The 2D black hole $\sigma$-model contains an operator that controls the world-sheet instanton contributions. This is given by a second screening operator in the world-sheet CFT, and the integration over the zero mode of the linear dilaton, once such a screening operator is considered, yields an $\alpha^{\prime}$-dependent function of the string states momenta. Therefore, it is natural to ask to what extent such an $\alpha^{\prime}$-dependent function accounts for the high-energy phase shift discussed in [1]. We will study this in Sect. 5. Before, in Sect. 2, we review the 2D Euclidean black hole $\sigma$ model. In Sect. 3, we discuss string scattering amplitudes on the black hole background and the high-energy modification of the phase shift. In Sect. 4, we review how such a modification is gathered in the FZZ dual theory. In Sect. 5, we first show how the second screening is associated to world-sheet instantons, and then we study their contribution in relation to the stringy phase shift.

\section{String 2D black hole}

String theory admits an interesting exact solution that describes strings propagating on a two-dimensional black hole background $[3,4]$. The metric of the classical Euclidean black hole geometry is given by

$\mathrm{d} s^{2}=L^{2}\left(\mathrm{~d} r^{2}+\tanh ^{2}(r) \mathrm{d} \theta^{2}\right)$, 
where $\theta \in[0,2 \pi)$ and $r \in \mathbb{R}_{>0}$. On the other hand, the dilaton configuration needed to support this background is given by

$\Phi(r)=\Phi_{0}-\log (\cosh (r))$,

which tends linearly to infinity when $r$ is large, namely far from the horizon. The metric above describes a semi-infinite cigar-like geometry of the $\mathbb{R}^{2}$ topology. The compact coordinate $\theta$ represents the compactified Euclidean time. The horizon of the Lorentzian black hole gets mapped to the tip of the cigar, which is located at $r=0$. In the large $r$ region, the Euclidean metric approaches a cylinder of radius $L$.

The non-linear $\sigma$-model that describes 2D string theory propagating on the background (1) and (2) is given by the gauged Wess-Zumino-Witten (WZW) model for the coset $H_{3}^{+} / U(1)$, with $H_{3}^{+}=S L(2, \mathbb{C}) / S U(2)$. Its Lorentzian version corresponds to the $S L(2, \mathbb{R}) / U(1)$ coset [4]. Let us first discuss the $\sigma$-model on $\mathrm{H}_{3}^{+}$, which in a convenient coordinate system takes the form

$$
\begin{aligned}
S_{M}= & \frac{L^{2}}{2 \pi} \int \mathrm{d}^{2} z\left(\partial \phi \bar{\partial} \phi+\beta \bar{\partial} \gamma+\bar{\beta} \partial \bar{\gamma}-\frac{R \phi}{2 \sqrt{2(k-2)}}\right. \\
& \left.-2 \pi M \beta \bar{\beta} \mathrm{e}^{-\sqrt{\frac{2}{k-2}} \phi}\right)
\end{aligned}
$$

which involves a scalar field $\phi$ and a $\beta-\gamma$ commuting ghost system. At large $r$, one can identify $\phi \sim \sqrt{k} r$, and this corresponds to a linear dilaton background. The constant $M$ appearing in (3) can be shown to be related to the twodimensional black hole mass. Its precise value is not relevant for perturbative physics, as it can be set to 1 by simply rescaling $\phi$. The relevant parameter is actually

$k=\frac{L^{2}}{\alpha^{\prime}}$,

which is the Reynolds number that controls the physics of the problem: It measures the typical strings length, $l_{s}=\sqrt{\alpha^{\prime}}$, relative to the curvature radius of the geometry, $L$. The classical limit thus corresponds to $k$ going to infinity.

Ghost fields $\beta$ and $\bar{\beta}$ in (3) are not dynamical, and then they can be integrated out. However, it is convenient to keep these fields in the action for practical purposes. One of the reasons for doing this is that it allows for a free-field representation of the $\operatorname{sl}(2, \mathbb{R})_{k} \oplus \operatorname{sl}(2, \mathbb{R})_{k}$ Kac-Moody symmetry that the action (3) exhibits. This free-field realization is given by the so-called Wakimoto representation of the $s l(2, \mathbb{R})_{k}$ current algebra, which follows from defining the local currents [5]

$J^{+}(z)=\beta(z)$,

$J^{3}(z)=-\beta(z) \gamma(z)-\sqrt{\frac{k-2}{2}} \partial \phi(z)$,

$J^{-}(z)=\beta(z) \gamma^{2}(z)+\sqrt{2 k-4} \gamma(z) \partial \phi(z)+k \partial \gamma(z)$, together with their complex conjugate counterpart. Considering the free-field propagators both for the scalar field $\phi$ and for the $\beta-\gamma$ system, the operator product expansion (OPE) of currents (4)-(6) realizes the $s l(2, \mathbb{R})_{k}$ affine Kac-Moody algebra. It can be verified that the interaction term in (3) has a regular OPE with the currents, so it preserves the full $s l(2, \mathbb{R})_{k}$ symmetry.

Through the Sugawara construction, the currents (4)-(6) yield the stress tensor

$T_{S L(2, \mathbb{R})}=\beta(z) \partial \gamma(z)-\frac{1}{2}(\partial \phi(z))^{2}-\frac{1}{\sqrt{2(k-2)}} \partial^{2} \phi(z)$,

together with its anti-holomorphic counterpart.

The coset $S L(2, \mathbb{R}) / U(1)$ construction can be accomplished by supplementing the $S L(2, \mathbb{R})$-model by adding an extra scalar field $X(z)=X_{\mathrm{L}}(z)+X_{\mathrm{R}}(\bar{z})$ and a fermionic $B-C$ ghost system [6-8]. This amounts to improve the stress tensor (7) with an extra piece, namely ${ }^{1}$

$T_{S L(2, \mathbb{R}) / U(1)}=T_{S L(2, \mathbb{R})}-B(z) \partial C(z)-\frac{1}{2}(\partial X(z))^{2}$,

and analogously for the anti-holomorphic counterpart. This yields the central charge

$c=\frac{2 k+2}{k-2}$,

which consistently tends to 2 in the large $k$ limit.

The BRST charge associated to the $U(1)$ of the coset model is

$Q_{\mathrm{BRST}}^{U(1)}=\int \mathrm{d} z C(z)\left(J^{3}(z)-i \sqrt{k / 2} \partial X(z)\right)$.

This means that the vertex operators creating physical states of the theory have to have regular OPE with the current $J^{3}-$ $i \sqrt{k / 2} \partial X$. Such operators are

$$
\begin{aligned}
V_{j, m, \bar{m}}(z, \bar{z})= & \gamma^{j-m}(z) \bar{\gamma}^{j-\bar{m}}(\bar{z}) \\
& \times \mathrm{e}^{\sqrt{\frac{2}{k-2} j \phi(z, \bar{z})}} \mathrm{e}^{i \sqrt{\frac{2}{k}}\left(m X_{\mathrm{L}}(z)+\bar{m} X_{\mathrm{R}}(\bar{z})\right)},
\end{aligned}
$$

where $j, m$, and $\bar{m}$ are isospin variables that label the $S L(2, \mathbb{R}) \times S L(2, \mathbb{R})$ representations. These variables represent the momenta associated to the radial and Euclidean time coordinates and the winding number along the latter. More precisely, we have the radial momentum

$p_{\phi} \equiv-i \frac{2 j+1}{\sqrt{k-2}}$

\footnotetext{
${ }^{1}$ Hereafter we omit the diffeomorphism $b-c$ ghost system contributions.
} 
and the right- and left-moving momenta

$$
\begin{aligned}
& p_{\mathrm{L}} \equiv \frac{2}{\sqrt{k}} m=\left(\omega \sqrt{k}+p_{\theta}\right), \\
& p_{\mathrm{R}} \equiv \frac{2}{\sqrt{k}} \bar{m}=\left(\omega \sqrt{k}-p_{\theta}\right),
\end{aligned}
$$

where $p_{\theta}$ and $\omega$ represent the momentum and the winding number along the $\theta$-direction, respectively. That is to say, the vertices (10) go like $V \sim \mathrm{e}^{\frac{i}{\sqrt{2}}\left(p_{\phi}-i Q\right) \phi} \mathrm{e}^{\frac{i}{\sqrt{2}}\left(p_{\mathrm{L}} X_{\mathrm{L}}+p_{\mathrm{R}} X_{\mathrm{R}}\right)}$, with $Q=-1 / \sqrt{k-2}$.

States with $p_{\phi} \in \mathbb{R}$ and $m-\bar{m} \in \mathbb{Z}$ correspond to vectors of the continuous series representation of $S L(2, \mathbb{R})$. Discrete representations have $j \in \mathbb{R}, m-\bar{m} \in \mathbb{Z}$ and $m+\bar{m}-j \in \mathbb{Z}$. Here, we will be involved with the former.

\section{High-energy scattering}

Now, let us discuss string scattering amplitudes on the background (1) and (2). Tree-level string scattering amplitudes are given by the integral of correlation functions of vertex operators on the sphere topology

$$
\begin{aligned}
A_{m_{1} m_{2} \ldots m_{N}}^{j_{1} j_{2} \ldots j_{N}}= & \int \prod_{i=1}^{N} \mathrm{~d}^{2} z_{i} \operatorname{Vol}_{S L(2, \mathbb{C})}^{-1} \\
& \times\left\langle\prod_{\ell=1}^{N}: V_{j_{\ell}, m_{\ell}, \bar{m}_{\ell}}\left(z_{\ell}, \bar{z}_{\ell}\right):\right\rangle_{M}
\end{aligned}
$$

where $\operatorname{Vol}_{S L(2, \mathbb{C})}^{-1}$ is the volume of the conformal Killing group. The subscript $M$ in (14) refers to the fact that expectation value is defined by interacting action (3). More precisely, we can write

$$
\begin{aligned}
A_{m_{1} m_{2} \ldots m_{N}}^{j_{1} j_{2} \ldots j_{N}}= & \int \prod_{i=4}^{N} \mathrm{~d}^{2} z_{i} \int \mathcal{D}^{2} \gamma \mathcal{D}^{2} \beta \mathcal{D} \phi \mathcal{D} X \mathrm{e}^{-S_{M}} \\
& \times \prod_{\ell=1}^{N} V_{j_{\ell}, m_{\ell}, \bar{m}_{\ell}}\left(z_{\ell}, \bar{z}_{\ell}\right)
\end{aligned}
$$

where the world-sheet insertions of the three vertices are fixed as $z_{1}=\bar{z}_{1}=0, z_{2}=\bar{z}_{2}=0$, and $z_{3}=\bar{z}_{3}=\infty$ in order to cancel the stabilized factor $\operatorname{Vol}_{S L(2, \mathbb{C})}^{-1}$. The functional measures $\mathcal{D}^{2} \gamma$ and $\mathcal{D}^{2} \beta$ stand for both the holomorphic and anti-holomorphic contributions of the ghost system. It can be shown that the integration on $\beta$ and $\bar{\beta}$ is what ultimately induces the one-loop corrections in (3), including in particular the linear dilaton term.

To perform the functional integral (15) it is convenient to first separate the zero mode $\phi_{0}$ of the field $\phi$. That is, consider $\phi(z, \bar{z})=\phi_{0}+\tilde{\phi}(z, \bar{z})$ and then integrate over the dilaton zero mode $\phi_{0}$ and fluctuations $\tilde{\phi}(z, \bar{z})$ separately. When doing this, one verifies that the integrand in (15) involves a contribution

$$
\begin{aligned}
& \mathrm{e}^{-\tilde{S}_{0}} \mathrm{e}^{\sqrt{\frac{2}{k-2}} j_{i} \tilde{\phi}\left(z_{i}, \bar{z}_{i}\right)} \int_{\mathbb{R}} \mathrm{d} \phi_{0} \int_{\mathbb{R}_{+}} \mathrm{d} \eta \delta\left(\eta-\mathrm{e}^{-\phi_{0} \sqrt{\frac{2}{k-2}}} \tilde{S}_{\mathrm{I}}\right) \\
& \times \mathrm{e}^{-\eta} \mathrm{e}^{\phi_{0} \sqrt{\frac{2}{k-2}}\left(\sum_{i=1}^{N} j_{i}+\frac{1}{4 \pi} \int \mathrm{d}^{2} z R\right),}
\end{aligned}
$$

which comes from separating the action (3) in its free part $S_{0} \equiv S_{M=0}$ and the interaction term

$S_{\mathrm{I}}=M \int \mathrm{d}^{2} z W(z, \bar{z}), \quad W=\beta(z) \bar{\beta}(\bar{z}) \mathrm{e}^{-\sqrt{\frac{2}{k-2}} \phi(z, \bar{z})}$.

In (16), quantities with tilde, such as $\tilde{S}_{0}$ and $\tilde{S}_{\mathrm{I}}$, are defined by replacing the field $\phi(z, \bar{z})$ by its fluctuations $\tilde{\phi}(z, \bar{z})$, e.g. $S_{0}=\tilde{S}_{0}$ and $S_{\mathrm{I}}=\mathrm{e}^{-\phi_{0} \sqrt{\frac{2}{k-2}}} \tilde{S}_{\mathrm{I}}$. Considering the GaussBonnet theorem, which states that the Euler characteristic on the sphere is $\frac{1}{2 \pi} \int \mathrm{d}^{2} z \sqrt{g} R=2$, and using properties of the $\delta$-function, one finds that, after integrating over $\phi_{0},(16)$ takes the form

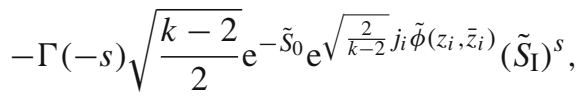

where $s=\sum_{i=1}^{N} j_{i}+1$ and where $\Gamma(-s)=\int_{\mathbb{R}_{+}} \mathrm{d} \eta \eta^{-1-s} \mathrm{e}^{-\eta}$. Finally, absorbing a $k$-dependent factor in the definition of the path integral, one finds

$$
\begin{aligned}
A_{m_{1} m_{2} \ldots m_{N}}^{j_{1} j_{2} \ldots j_{N}}= & \Gamma(-s) M^{s} \int \prod_{i=4}^{N} \mathrm{~d}^{2} z_{i} \int \prod_{r=1}^{s} \mathrm{~d}^{2} w_{r} \\
& \times F_{m_{1} m_{2} \ldots m_{N}}^{j_{1} j_{2} \ldots j_{N}}\left(z_{1}, \ldots z_{N}, w_{1}, \ldots w_{s}\right)
\end{aligned}
$$

with

$$
\begin{aligned}
& F_{m_{1} m_{2} \ldots m_{N}}^{j_{1} j_{2} \ldots j_{N}} \\
& \quad=\left\langle\prod_{i=1}^{N}: V_{j_{i}, m_{i}, \bar{m}_{i}}\left(z_{i}, \bar{z}_{i}\right): \prod_{r=1}^{s}: W\left(w_{r}, \bar{w}_{r}\right):\right\rangle_{M=0}
\end{aligned}
$$

where now the expectation value (20) is defined with respect to the free action $S_{0}$. This permits to perform the Wick contractions resorting to the free-field propagators. The insertion of the $s$ additional integrated vertices $M \int \beta \bar{\beta} \mathrm{e}^{-\sqrt{\frac{2}{k-2}} \tilde{\phi}}$ in (20) comes from the factor $\left(\tilde{S}_{\mathrm{I}}\right)^{s}$ in (18). Recall we have

$s=1+\sum_{i=1}^{N} j_{i}$

Then we see that the integration over the dilaton zero mode is responsible for the prefactor $\Gamma(-s)$ in (19). This prefactor is crucial for our discussion. This produces poles at configurations $s=1+\sum_{i=1}^{N} j_{i} \in \mathbb{Z}_{\geq 0}$, whose residues can be thought of as resonant correlators. The poles $s \in \mathbb{Z}_{>0}$ admit a physical interpretation similar to that proposed in Ref. [9] for the analogous poles in Liouville field theory. 
Formula (19) can also be interpreted within the context of the Coulomb gas realization of 2D CFT correlation functions, where the insertion of $s$ operators $\tilde{S}_{\text {I }}$ correspond to the inclusion of screening charges needed to satisfy the charge condition imposed by the presence of the background charge $Q=-1 / \sqrt{k-2}$ at infinity.

The black hole mass parameter $M$ in the amplitudes (19) plays the role of string coupling constant: Its power depends on the genus of the surface goes like $M^{s+1-g}$ and, as mentioned above, its absolute value is determined by the zero mode of the dilaton, i.e. $M \sim \mathrm{e}^{\Phi_{0}}$.

When integrating over the $\beta-\gamma$ system, and because $\beta$ is a 1-differential, the Riemann-Noch theorem, once combined with (21), yields exactly the same conservation law as obtained from the integration over the zero mode of the field $X$, namely $\sum_{i=1}^{N}\left(m_{i}+\bar{m}_{i}\right)=0$. The condition on the total winding number $\sum_{i=1}^{N}\left(m_{i}-\bar{m}_{i}\right)$, on the other hand, is more subtle $[10,11]$.

The prefactor $\Gamma(-s)$ in (19) can be alternatively obtained by virtually integrating over the imaginary part of $\phi_{0}$. This produces a $\delta$-function that selects a precise amount of operators $\tilde{S}_{\mathrm{I}}$ from the series expansion of $\mathrm{e}^{-S_{\mathrm{I}}}$. More precisely, the $\delta$-function selects the $s$ th term $\frac{(-1)}{s !}\left(S_{\mathrm{I}}\right)^{s}$ of the series, with $s=\sum_{i=1}^{N} j_{i}+1$. Then the infrared divergence $\delta(0) \sim \Gamma(0)$ can be combined with the multiplicity factor $(-1) / s$ ! in order to produce the factor $\Gamma(-s)$ by recalling the formula $\lim _{\varepsilon \rightarrow 0} \Gamma(-s+\varepsilon) / \Gamma(\varepsilon)=(-1)^{s} / \Gamma(s+1)$ for $s \in \mathbb{Z}_{\geq 0}$.

Correlation functions (19) and (20) can be explicitly computed for the cases $N=2$ and $N=3$. The expression for the two-point function is [8]

$$
\begin{aligned}
A_{m-m}^{j j}= & \left(-\pi M \frac{\Gamma\left(\frac{1}{k-2}\right)}{\Gamma\left(\frac{k-3}{k-2}\right)}\right)^{2 j+1} \frac{\Gamma\left(1-\frac{2 j+1}{k-2}\right)}{\Gamma\left(1+\frac{2 j+1}{k-2}\right)} \\
& \times \frac{\Gamma(1+j-m) \Gamma(1+j+\bar{m}) \Gamma(-2 j)}{\Gamma(-j-m) \Gamma(-j+\bar{m}) \Gamma(2 j+1)} .
\end{aligned}
$$

The phase shift $\delta$ is defined by the reflection coefficients, given by the two-point function as $\mathrm{e}^{i \delta} \equiv A_{m}^{j}{ }^{j}{ }_{-m}$. Since $M$ can be adjusted to absorb the $k$-dependent functions in the first factor of (22), the only relevant $k$-dependent piece in the two-point function is given by the prefactor

$\frac{\Gamma\left(1-\frac{2 j+1}{k-2}\right)}{\Gamma\left(1+\frac{2 j+1}{k-2}\right)}=\Gamma\left(1-i \frac{p_{\phi}}{\sqrt{k-2}}\right) \Gamma^{-1}\left(1+i \frac{p_{\phi}}{\sqrt{k-2}}\right)$.

This prefactor is the one responsible for the phaseshift modification at high energy discussed in [1]. To see this, following [1], one may resort to the Stirling approx- imation, which states that for large $|z|$ one has $\Gamma(z) \simeq$ $\sqrt{2 \pi / z}(z / e)^{z}(1+\mathcal{O}(1 / z))$. Applying this to (23), one finds that in the regime $p_{\phi} \gg \sqrt{k-2}$, one finds

$\mathrm{e}^{i \delta} \simeq-\mathrm{e}^{-\frac{2 i p_{\phi}}{\sqrt{k-2}}\left(\log p_{\phi}-1-\frac{1}{2} \log (k-2)\right)} \mathrm{e}^{\frac{i \pi}{2}}$.

This gives the high-energy dependence of the phase shift [1].

\section{The dual theory}

The two-dimensional $\sigma$-model on the Euclidean 2D black hole geometry is well known to be dual to a two-dimensional conformal field theory known as sine-Liouville theory. The latter theory is defined by the action

$$
\begin{aligned}
S_{\lambda}= & \frac{1}{2 \pi} \int \mathrm{d}^{2} z\left(\partial \varphi \bar{\partial} \varphi+\partial X \bar{\partial} X-\frac{R \varphi}{2 \sqrt{2 k-4}}\right. \\
& \left.+2 \pi \lambda \mathrm{e}^{-\sqrt{\frac{k-2}{2}} \varphi} \cos (\sqrt{k / 2} \tilde{X})\right)
\end{aligned}
$$

where $\tilde{X}(z, \bar{z}) \equiv X_{\mathrm{L}}(z)-X_{\mathrm{R}}(\bar{z})$ is the dual to the bosonic field $X(z, \bar{z})=X_{\mathrm{L}}(z)+X_{\mathrm{R}}(\bar{z})$. The direction $X$ is compact, while $\varphi$ takes values on the real line. That is to say, unlike the 2D Euclidean black hole $\sigma$-model, the model defined by (25) has the topology $\mathbb{R} \times S^{1}$. Thought of as a string $\sigma$-model action, sine-Liouville theory (25) represents a flat linear dilaton background in the presence of a nonhomogeneous tachyon condensate.

The duality between (3) and (25) has been conjectured by Fateev, Zamolodchikov, and Zamolodchikov (FZZ) in an unpublished work [2], and it has been reviewed and elaborated by Kazakov, Kostov, and Kutasov in Ref. [12]. It represents a kind of T-duality. In fact, the supersymmetric version of the FZZ duality actually corresponds to mirror symmetry [13], which relates the $\mathcal{N}=2$ version of Liouville theory with the Kazama-Susuki $S L(2, \mathbb{R}) / U(1)$-model. In [14,15], Maldacena explained how the bosonic FZZ duality emerges as a consequence of the supersymmetric extension. More recently, a proof of the FZZ conjectured duality was given by Hikida and Shomerus in Ref. [16]; see also [17].

FZZ duality is a statement about the identity of correlation functions of both models. It states that correlation functions (19) and (20) (or, equivalently, (32) and (33)) coincide with the sine-Liouville correlation functions involving the vertex operators

$$
V_{j, m, \bar{m}}(z, \bar{z})=\mathrm{e}^{\sqrt{\frac{2}{k-2}} j \varphi(z, \bar{z})} \mathrm{e}^{i \sqrt{\frac{2}{k}}\left(m X_{\mathrm{L}}(z)+\bar{m} X_{\mathrm{R}}(\bar{z})\right)} .
$$


The interaction term in the sine-Liouville action can actually be written as particular cases of (26), namely

$$
\begin{aligned}
& \lambda \int \mathrm{d}^{2} z \mathrm{e}^{-\sqrt{\frac{k-2}{2}} \varphi} \cos (\sqrt{k / 2} \tilde{X})=\frac{\lambda}{2} \int \mathrm{d}^{2} z V_{1-\frac{k}{2}, \frac{k}{2},-\frac{k}{2}} \\
& +\frac{\lambda}{2} \int \mathrm{d}^{2} z V_{1-\frac{k}{2},-\frac{k}{2}, \frac{k}{2}} .
\end{aligned}
$$

Correlation functions involving (26) were computed in Ref. [18] using the Coulomb gas approach. The correlators are defined by inserting $s_{+}$operators of the type $\frac{\lambda}{2} \int \mathrm{d}^{2} z \quad V_{1-k / 2, k / 2,-k / 2}$ and $s_{-}$operators of the type $\frac{\lambda}{2} \int \mathrm{d}^{2} z V_{1-k / 2,-k / 2, k / 2}$, satisfying the condition

$\sum_{i=1}^{N} j_{i}+1=\frac{k-2}{2}\left(s_{+}+s_{-}\right), \quad$ with $\quad s_{ \pm} \in \mathbb{Z}$.

In this theory, the total winding number $\sum_{i=1}^{N} \omega_{i}=$ $\sum_{i=1}^{N}\left(m_{i}-\bar{m}_{i}\right) / k$ in a given $N$-point function can be violated up to $N-2$ units [2]. The winding number preserving correlation functions correspond to the particular cases $s_{+}=s_{-}=\left(\sum_{i=1}^{N} j_{i}+1\right) /(k-2)$. On the other hand, correlators with $\sum_{i=1}^{N} \omega_{i} \neq 0$ correspond to correlators computed with $s_{+}-s_{-} \neq 0$, so that the quantity $\left(\sum_{i=1}^{N} j_{i}+1\right) /(k-2)$ in the latter case is not necessarily an integer number. In the case of the two-point function $(N=2)$ the winding number is preserved, and thus $s_{-}=s_{+}$. This implies that one can describe the sine-Liouville 2-point correlation functions by inserting $s_{+}+s_{-}$operators $\mathrm{e}^{-\sqrt{\frac{k-2}{2}} \varphi} \cos (\sqrt{k / 2} \tilde{X})$. It can be shown [12] that, in this case, the integration over the zero mode of $\varphi$ in the sine-Liouville two-point function yields a factor $^{2}$

$$
\begin{aligned}
\Gamma\left(-2 \frac{2 j+1}{k-2}\right) \simeq & \sqrt{2 \pi} \mathrm{e}^{-\frac{2 i p_{\phi}}{\sqrt{k-2}}\left(\log p_{\phi}-1+\log 2-\frac{1}{2} \log (k-2)\right)} \\
& \times \mathrm{e}^{-\frac{\pi p_{\phi}}{\sqrt{k-2}}} \mathrm{e}^{-\frac{1}{2} \log \left(\frac{2 p_{\phi}}{\sqrt{k-2}}\right)} \mathrm{e}^{\frac{i \pi}{4}}
\end{aligned}
$$

and, as observed in [1], this factor precisely reproduces the leading piece $\sim p_{\phi} \log p_{\phi}$ of the phase shift ${ }^{3}$ (24). In addition, it was observed there that the integral over the zero mode of $\varphi$, which in the case of sine-Liouville theory produces the $\Gamma$-function (28), receives dominant contributions coming from a region of the moduli space that, in the $2 \mathrm{D}$ Euclidean black hole $\sigma$-model side, has no representative. This suggests that sine-Liouville description is the appropriate one to describe these finite- $\alpha^{\prime}$ effects. To complete the argument, in the next section we will address the following two questions: First, whether (and how) finite- $\alpha^{\prime}$ effects

\footnotetext{
${ }^{2}$ Here, the symbol $\simeq$ means that this has to be understood as valid in the limit $p_{\phi}>>\sqrt{k-2}$.

3 Although the coefficient of the term in $\delta$ that is linear in $p_{\phi}$ differs from that in (24).
}

(finite- $k$ effects) can be gathered in the 2D Euclidean black hole $\sigma$-model by the integration over $\phi_{0}$. Secondly, whether (or to what extent) such $\alpha^{\prime}$ effects recover the phase shift (24).

\section{World-sheet instantons}

The conformal field theory defined by the Kac-Moody currents (4)-(6) and the stress tensor (8) admits another local exactly marginal operator. This is given by

$$
\begin{aligned}
& S_{\mathrm{II}}=\tilde{M} \int \mathrm{d}^{2} z \tilde{W}(z, \bar{z}), \\
& \tilde{W}=W^{k-2}=(\beta(z) \bar{\beta}(\bar{z}))^{k-2} \mathrm{e}^{-\sqrt{2(k-2)} \phi(z, \bar{z})} .
\end{aligned}
$$

Indeed, one can verify that the OPE between (29) and currents (4)-(6) is regular, up to total derivatives. In particular, it yields

$$
\begin{aligned}
& J^{-}(z) \beta^{k-3}(w) \mathrm{e}^{-\sqrt{2(k-2)} \phi(w)} \times \text { h.c. } \\
& \simeq \partial_{w}\left(\frac{k-2}{z-w} \beta^{k-3}(w) \mathrm{e}^{-\sqrt{2(k-2)} \phi(w)}\right) \times \text { h.c. }+\cdots
\end{aligned}
$$

where h.c. stands for the Hermitian conjugate contribution. Operator (29) commutes with the BRST charge (9), and has dimension 1 with respect to the stress tensor (8). Then one can in principle consider operators $S_{\text {II }}$ as the screening operator to be used to define the correlation functions. From the two-dimensional conformal field theory point of view, the inclusion of operators (29) is completely natural, as it corresponds to a suitable screening charge in the Coulomb gas representation of 2D CFT correlation functions. However, within the context of the path integral approach, their inclusion is less clear as their interpretation in the world-sheet string $\sigma$-model is neither that of a graviton nor of a tachyon condensate. We will argue below that operators (29) are actually associated to a type of world-sheet instantons discussed in Ref. [11].

If one includes both operators (17) and (29) in the correlators, then one finds the following condition for the observables not to vanish:

$s+\tilde{s}(k-2)=1+\sum_{i=1}^{N} j_{i}$

where $s$ and $\tilde{s}$ are the amount of operators of the type $S_{\mathrm{I}}$ and of the type $S_{\mathrm{II}}$, respectively.

It was proven in [10] that the correlation functions (15) computed with $s$ screening operators of the type (17) and no operators of the type (29) exactly agree with the correlation functions computed by inserting $\tilde{s}=s /(k-2)$ operators of the type (29) and no operators of the type (17) provided the couplings $M$ and $\tilde{M}$ are related by 


$$
\begin{aligned}
& \tilde{M}=c_{k} M^{k-2}, \quad \text { with } \\
& c_{k}=\pi^{k-3} \frac{\Gamma(k-1)}{\Gamma(-k+2)}\left(\frac{\Gamma\left(\frac{1}{k-2}\right)}{\Gamma\left(\frac{k-3}{k-2}\right)}\right)^{k-2} .
\end{aligned}
$$

This means that we can write amplitudes (19) and (20) as follows:

$$
\begin{aligned}
& A_{m_{1} m_{2} \ldots m_{N}}^{j_{1} j_{2} \ldots j_{N}}=\Gamma(-\tilde{s}) c_{k}^{\tilde{s}} M^{s} \int \prod_{i=4}^{N} \mathrm{~d}^{2} z_{i} \\
& \quad \times \int \prod_{r=1}^{\tilde{s}} \mathrm{~d}^{2} w_{r} \tilde{F}_{m_{1} m_{2} \ldots m_{N}}^{j_{1} j_{2} \ldots j_{N}}\left(z_{1}, \ldots z_{N} ; w_{1}, \ldots w_{s}\right)
\end{aligned}
$$

where

$$
\begin{aligned}
& \tilde{F}_{m_{1} m_{2} \ldots m_{N}}^{j_{1} j_{2} \ldots j_{N}} \\
& \quad=\left\langle\prod_{i=1}^{N}: V_{j_{i}, m_{i}, \bar{m}_{i}}\left(z_{i}, \bar{z}_{i}\right): \prod_{r=1}^{\tilde{s}}: \tilde{W}\left(w_{r}, \bar{w}_{r}\right):\right\rangle_{M=0}
\end{aligned}
$$

and where the amount of screening operators is given by

$$
\tilde{s}=\frac{1}{(k-2)}\left(1+\sum_{i=1}^{N} j_{i}\right),
$$

where the prefactor $\Gamma(-\tilde{s})$ in $(32)$, which has an origin completely analogous to the factor $\Gamma(-s)$ in (19), now exhibits poles at

$1+\sum_{i=1}^{N} j_{i}=n(k-2), \quad n \in \mathbb{Z}_{\geq 0}$.

Before discussing this prefactor in relation to the phase shift, let us discuss condition (35) in relation to the worldsheet instantons. Condition (35) for $n=1$, in the case of the $\mathrm{N}$-point function, yields the pole condition

$1+\sum_{i=1}^{N} j_{i}=(k-2)$,

which in the convention of [11], which is related to ours through the simple changes of variables $j_{i} \rightarrow \hat{j}_{i}=j_{i}+1$, and for $n=1$ corresponds to

$\sum_{i=1}^{N} \hat{j}_{i}=k+N-3, \quad n \in \mathbb{Z}_{\geq 0}$.

Interestingly, this is exactly the pole condition that Maldacena and Ooguri conjectured in [11] that would appear in the $S L(2, \mathbb{R})$ WZW $N$-point correlation functions. One confirms from (37) that such poles actually appear. In [11], these poles were interpreted as world-sheet instantons, corresponding to classical string configurations of momentum $j \sim k$ that can extend to the large $\phi$ region with no cost of energy. These configurations correspond to holomorphic maps $\gamma=\gamma(z)$, associated to classical solutions, which extend in the $\phi$ direction with no potential preventing them from going to $\phi=\infty$. When integrating over auxiliary fields $\beta$ and $\bar{\beta}$, one produces an effective potential $\int \mathrm{d}^{2} z \partial \bar{\gamma} \bar{\partial} \gamma \mathrm{e}^{\sqrt{\frac{2}{k-2}} \phi}$, which vanishes for configurations with $\bar{\partial} \gamma=0$ (as operators (17) and (29) do). These classical configurations are closely related to the long strings discussed in [19].

Poles (35) with $n \geq 1$ also admit an interpretation as world-sheet instantons. In [11], such divergences are discussed for the particular case of the two-point function, namely for

$\hat{j}=\frac{n}{2}(k-2)+\frac{1}{2}, \quad n \in \mathbb{Z}_{\geq 0}$,

and are understood as world-sheet instantons wrapping on $S^{2}$ parameterized by the projective plane variables $\gamma, \bar{\gamma}$.

Now, going back to the phase shift, we observe that for $n=1$ poles (38) (i.e. (37) for $N=2$ ) have their origin in the prefactor

$$
\begin{aligned}
\Gamma(-\tilde{s}) \simeq & \sqrt{2 \pi} \mathrm{e}^{-\frac{i p_{\phi}}{\sqrt{k-2}}\left(\log p_{\phi}-1-\frac{1}{2} \log (k-2)\right)} \mathrm{e}^{\frac{i \pi}{4}} \\
& \times \mathrm{e}^{-\frac{\pi p_{\phi}}{2 \sqrt{k-2}}} \mathrm{e}^{-\frac{1}{2} \log \frac{p_{\phi}}{\sqrt{k-2}}},
\end{aligned}
$$

of (32), which looks pretty much like the contribution that yields the stringy phase shift, cf. (3.3) in [1]. However, a closer look at (39) reveals that there is a factor 2 missing with respect to (24). That is, the factor $\Gamma(-\tilde{s})$, which comes from the integration over the dilaton zero mode once the second screening operator is introduced, yields a $k$-dependent function of the momenta that only accounts for one half of the leading order modification that the phase shift suffers at high energy. In turn, one concludes, with the authors of [1], that at high energy the physics of the problem is better described by the FZZ dual model, even if the operator that controls the world-sheet instantons are considered in the 2D Euclidean black hole $\sigma$-model CFT.

Acknowledgments Work partially funded by FNRS-Belgium (convention FRFC PDR T.1025.14 and convention IISN 4.4503.15), by the Communauté Française de Belgique through the ARC program and by a donation from the Solvay family. The support of CONICET, FNRS+MINCyT, FONDECyT and UBA through grants PIP 0595/13, BE 13/03, Fondecyt 1140155 and UBACyT 20020120100154BA, respectively, is greatly acknowledged. The Centro de Estudios Científi$\cos$ (CECs) is funded by the Chilean Government through the Centers of Excellence Base Financing Program of CONICYT-Chile.

Open Access This article is distributed under the terms of the Creative Commons Attribution 4.0 International License (http://creativecomm ons.org/licenses/by/4.0/), which permits unrestricted use, distribution, and reproduction in any medium, provided you give appropriate credit to the original author(s) and the source, provide a link to the Creative 
Commons license, and indicate if changes were made. Funded by SCOAP ${ }^{3}$.

\section{References}

1. A. Giveon, N. Itzhaki, D. Kutasov, arXiv:1502.03633

2. V. Fateev, A. Zamolodchikov, Al. Zamolodchikov, unpublished

3. G. Mandal, A. Sengupta, S. Wadia, Mod. Phys. Lett. A 6, 1685 (1991)

4. E. Witten, Phys. Rev. D 44, 314 (1991)

5. M. Wakimoto, Commun. Math. Phys. 111, 75 (1986)

6. M. Bershadsky, D. Kutasov, Phys. Lett. B 266, 345 (1991)

7. R. Dijkgraaf, H. Verlinde, E. Verlinde, Nucl. Phys. B 371, 269 (1992)
8. K. Becker, M. Becker, Nucl. Phys. B 418, 206 (1994)

9. P. Di Francesco, D. Kutasov, Nucl. Phys. B 375, 119 (1992)

10. G. Giribet, C. Núñez, JHEP 06, 010 (2001)

11. J.M. Maldacena, H. Ooguri, Phys. Rev. D 65, 106006 (2002)

12. V. Kazakov, I. Kostov, D. Kutasov, Nucl. Phys. B 622, 141 (2002)

13. K. Hori, A. Kapustin, JHEP 08, 045 (2001)

14. J.M. Maldacena, JHEP 09, 078 (2005)

15. J.M. Maldacena, Int. J. Geom. Methods Mod. Phys. 3, 1 (2006)

16. Y. Hikida, V. Schomerus, JHEP 10, 064 (2007)

17. G. Giribet, M. Leoni, Rep. Math. Phys. 61, 151 (2008)

18. T. Fukuda, K. Hosomichi, JHEP 09, 003 (2001)

19. N. Seiberg, E. Witten, JHEP 04, 017 (1999) 